\title{
Nonlinear optical organic-inorganic crystals: Synthesis, structural analysis, and verification of harmonic generation in tri-(o-Chloroanilinium Nitrate)
}

\author{
Hamza Athmani ${ }^{\mathrm{a}, \mathrm{b}}$, Christian Kijatkin ${ }^{\mathrm{c}, \mathrm{e}}$, Rim Benali-Cherif ${ }^{\mathrm{a}}$, Sébastien Pillet ${ }^{\mathrm{b}}$, Dominik \\ Schaniel $^{\mathrm{b}}$, Mirco Imlau ${ }^{\mathrm{c}, \mathrm{e}}$, Nourredine Benali-Cherif ${ }^{\mathrm{a}, \mathrm{d}} \&$ El-Eulmi Bendeif ${ }^{\mathrm{b}^{*}}$

\footnotetext{
${ }^{a}$ Laboratoire des Structures, Propriétés et Interactions Interatomiques, Université Abbes LaghrourKhenchela, 40000 Khenchela, Algeria,

${ }^{\mathrm{b}}$ Université de Lorraine, CNRS, CRM ${ }^{2}$, Nancy, France

${ }^{\mathrm{c}}$ School of Physics, Osnabrück University, 49076 Osnabrück, Germany

${ }^{\mathrm{d}}$ Ecole Nationale Polytechnique, département de Génie des Matériaux, 25000, Constantine, Algeria

${ }^{\mathrm{e}}$ Research Center for Cellular Nanoanalytics, CellNanOs, Osnabrück University, 49076 Osnabrück, Germany

Correspondence e-mail: el-eulmi.bendeif@univ-lorraine.fr
}

\begin{abstract}
Structural and non-linear optical (NLO) properties of a new anilinium hybrid crystal of chemical formula $\left[\left(\mathrm{C}_{6} \mathrm{H}_{7} \mathrm{NCl}^{+}, \mathrm{NO}_{3}^{-}\right)_{3}\right]$ have been investigated. The crystal structure was determined from single crystal X-ray diffraction measurements performed at a temperature of $100 \mathrm{~K}$ and shows that the compound crystalizes in a noncentrosymmetric space group (Pna2 $\left.{ }_{1}\right)$. The structural analysis was coupled to the Hirshfeld surfaces analysis to evaluate the contribution of the different intermolecular interactions to the formation of supramolecular assemblies in the solid state that obey nonlinear optical features. This analysis reveals that the studied compound is characterized by a three-dimensional network of hydrogen bonds and the main contributions are provided by the $\mathrm{O} \ldots \mathrm{H}, \mathrm{C} \ldots \mathrm{H}, \mathrm{H} \ldots \mathrm{H}$ and $\mathrm{Cl} \ldots \mathrm{H}$ interactions, which alone represent $\sim 85 \%$ of the total contributions to the Hirshfeld surfaces. It is noteworthy that the Halogen...H contributions are quite comparable to those of H...H contacts. The nonlinear optical properties were investigated by nonlinear diffuse femtosecond-pulse reflectometry and the obtained results were compared to those of the reference material $\mathrm{LiNbO}_{3}$. The hybrid crystals exhibit notable second (SHG) and third (THG) harmonic generation that confirms its polarity generated by the different intermolecular interactions. These measurements also highlight that the THG signal of the new anilinium compound normalized to its SHG counterpart is more pronounced than for $\mathrm{LiNbO}_{3}$.
\end{abstract}




\section{Introduction}

Organic-inorganic hybrid materials have been extensively investigated due to their remarkable versatile properties. Among others, anilinium derivatives have received considerable attention in recent years, due to their interesting applications as starting materials in multiple industrial and pharmaceutical processes (Below et al., 2004; Yunnikova et al., 2013). The anilinium halides are used as precursors in the synthesis of azo dyes, pigments, pesticides, insecticides, antioxidants and cosmetic products (Cao et al., 2010; Wang et al., 2010). The m-chloroaniline is for example used as a precursor for the production of the widely used antimicrobial and bactericide Chlorohexidine (Bayar et al., 2017). These compounds are also employed in the elaboration of optoelectronic devices in particular with a focus to non-linear optical (NLO) features. A pronounced NLO efficiency in these materials is correlated to the high polarizability of the $\pi$-conjugated molecules of the organic part and to the extended network of the intermolecular interactions between the organic cations and the inorganic anions, which promotes the charge transfer within the materials. On the other hand, these compounds are well investigated because of the ease of synthetic approaches, for their chemical and mechanical stability and their ability to crystallize in non-centrosymmetric structures appropriate for even order NLO applications. Although the development of anilinium derivatives systems continues to grow and being the main research topic of several research groups, a search in the Cambridge Structural Database CSD (ConQuest Version 1.22, 2018) (Allen, 2002) for crystal structures containing chloroanilinium molecules results in 37 hits with only seven compounds crystallizing in a non-centrosymmetric space groups. Based on our experience in the synthesis of anilinium derivatives hybrid compounds (Bendeif et al., 2005; Boutobba et al., 2010; Dadda et al., 2012; Takouachet et al., 2016) we prepared a new Chloroanilinium nitrate compound: tri(o-Chloroanilinium Nitrate): $\left[\left(\mathrm{C}_{6} \mathrm{H}_{7} \mathrm{NCl}^{+}, \mathrm{NO}_{3}{ }^{-}\right)_{3}\right]$ named hereafter $\mathrm{o}-\mathrm{ClAN}$. The combination of the organic anilinium with the inorganic planar $\mathrm{NO}_{3}{ }^{-}$anion is expected to yield enhanced NLO properties with respect to non-planar anions (Bouchouit et al., 2010) if crystallization in a non-centrosymmetric space group is achieved. 


\section{Experimental}

\subsection{Synthesis and crystallization}

Crystals of tri(o-chloroanilinium nitrate): $\left[\left(\mathrm{C}_{6} \mathrm{H}_{7} \mathrm{NCl}^{+}, \mathrm{NO}_{3}^{-}\right)_{3}\right]$ (Fig. 1) were obtained by adding nitric acid to an aqueous solution containing o-chloroaniline in a 1:1 stoichiometric ratio. After three weeks of slow evaporation at room temperature, small brown single crystals appeared. All chemicals (reagents and solvents) used for the synthesis were purchased from Sigma Aldrich, and used without further purification.

\subsection{Single crystal $X$-ray measurements}

The single crystal X-ray diffraction experiments were performed at $100 \mathrm{~K}$ on a SuperNova Dual Wavelength Microfocus diffractometer equipped with a $135 \mathrm{~mm}$ Atlas CCD detector, using Mo $\mathrm{K}_{\alpha}$ radiation $(\lambda=0.71073 \AA)$. To ensure high redundancies, the intensity data were accurately collected using $\omega$ oscillation scans of $1^{\circ}$ per frame repeated at different $\varphi$ positions. The radiation exposure time was $30 \mathrm{~s}$ per frame. The CrysAlis program suite (Rigaku Oxford Diffraction, 2017) was used for the unit cell determination, data reduction and analytical absorption corrections. The diffraction frames have been indexed and integrated yielding 117559 reflections up to a maximum resolution of $\sin \theta_{\max } / \lambda=0.864 \AA^{-1}$ and merged to 13028 unique reflections $\left(\mathrm{R}_{\text {int }}=0.072\right)$ with a coverage of reciprocal space of more than $99.6 \%$. The corresponding structure was solved in the space group $P-1$ by direct methods and successive Fourier difference syntheses and refined against $\mathrm{F}^{2}$ by weighted full-matrix least-squares methods using the SHELXL97 program (Sheldrick, 2008). All non-H atoms were refined anisotropically. All calculations were carried out using the WinGX software package (Farrugia, 1999).The crystallographic data, measurements and refinement details are summarized in Table 1.

\subsection{Hirshfeld surfaces calculations}

The Hirshfeld surface $(H S)$ is a useful concept for describing the influence of molecular interactions by partitioning the total electron density in the crystal into molecular fragments where the electron distribution of a sum of spherical atoms for the molecule dominates the corresponding sum over the crystal (Hirshfeld, 1977; Spackman \& Jayatilaka., 2009). Thus, the $H S$ can be considered as the boundaries delimiting the space occupied by the molecular electron density from those of its neighboring molecules in a crystal. The size and shape of the $H S$ also allow qualitative and quantitative investigations as well as the visualization of the different intermolecular interactions. The $H S$ presented in this work were calculated with the program Crystal Explorer (Wolff et al., 2012) and are mapped using the normalized contact distance $\left(d_{\text {norm }}\right)$, which is calculated using the following formula:

$$
d_{n o r m}=\frac{d_{i}-r_{i}^{v d W}}{r_{i}^{v d W}}+\frac{d_{e}-r_{e}^{v d W}}{r_{e}^{v d W}}
$$

where $d_{\text {norm }}$ represent the sum of the distances of any surface point to the nearest external $\left(d_{e}\right)$ and internal $\left(d_{i}\right)$ atoms, normalized by the Van der Waals radii of the atoms. It is also worth 
noting that $H S$ maps are characterized by a red-blue-white color scheme: red regions represent closer contacts and negative $d_{\text {norm }}$ value; blue regions represent longer contacts and positive $d_{\text {norm }}$ value; and white regions correspond to interactions with bond lengths exactly equivalent to the Vander Waals separation and with a $d_{\text {norm }}$ value of zero.

From the 3-D $d_{\text {norm }}$ surface, we can obtain 2-D fingerprint plots, allowing easy attribution and quantitative analysis of the contribution of all intermolecular contacts at the same time. Thus, these $2 \mathrm{D}$ plots summarize the nature and type as well as the contributions of all intermolecular interactions.

Table 1: Crystallographic and refinement details for the structures of tri(o-chloroanilinium nitrate) salt.

\begin{tabular}{|c|c|}
\hline & tri(o-chloroanilinium nitrate) \\
\hline \multicolumn{2}{|l|}{ Crystal data } \\
\hline Chemical formula & $\left(\mathrm{C}_{6} \mathrm{H}_{7} \mathrm{ClN}^{+} . \mathrm{NO}_{3}{ }^{-}\right)_{3}$ \\
\hline $\mathrm{M} r$ & 571.76 \\
\hline $\begin{array}{l}\text { Crystal system, space } \\
\text { group }\end{array}$ & Orthorhombic, Pna $2_{1}$ \\
\hline Temperature $(\mathrm{K})$ & 100 \\
\hline$a, b, c(\AA)$ & $16.8015(5), 6.5945$ (2), $22.4243(6)$ \\
\hline $\mathrm{V}(\AA 3)$ & $2484.56(12)$ \\
\hline $\mathrm{Z}$ & 4 \\
\hline Radiation type & Mo K $\alpha$ \\
\hline$\mu\left(\mathrm{mm}^{-1}\right)$ & 0.43 \\
\hline Crystal size $(\mathrm{mm})$ & $0.1 \times 0.05 \times 0.1$ \\
\hline \multicolumn{2}{|l|}{ Data collection } \\
\hline Diffractometer & Oxford Agilent-SuperNova \\
\hline $\begin{array}{l}\text { No. of measured, } \\
\text { independent and observed } \\
\text { with }[\mathrm{I}>2 \sigma(\mathrm{I})] \text { reflections }\end{array}$ & $117559,13028,9377$ \\
\hline Rint & 0.072 \\
\hline$(\sin \theta / \lambda) \max \left(\AA^{-1}\right)$ & 0.864 \\
\hline \multicolumn{2}{|l|}{ Refinement } \\
\hline$R[F 2>2 \sigma(F 2)], w R(F 2), S$ & $0.047,0.099,1.07$ \\
\hline No. of reflection & 13028 \\
\hline No. of parameters & 409 \\
\hline No. of restraints & 1 \\
\hline H-atom treatment & All $\mathrm{H}$-atom coordinates parameters refined \\
\hline$\Delta \rho \max , \Delta \rho \min (\mathrm{e} \AA-3)$ & $0.45,-0.38$ \\
\hline Absolute structure & $\begin{array}{l}\text { Flack x determined using } 3510 \text { quotients [(I+)-(I- } \\
)] /[(\mathrm{I}+)+(\mathrm{I}-)] \text { (Parsons, Flack and Wagner, Acta } \\
\text { Cryst. B69 (2013) 249-259). }\end{array}$ \\
\hline $\begin{array}{l}\text { Absolute structure } \\
\text { parameter }\end{array}$ & $-0.050(16)$ \\
\hline
\end{tabular}




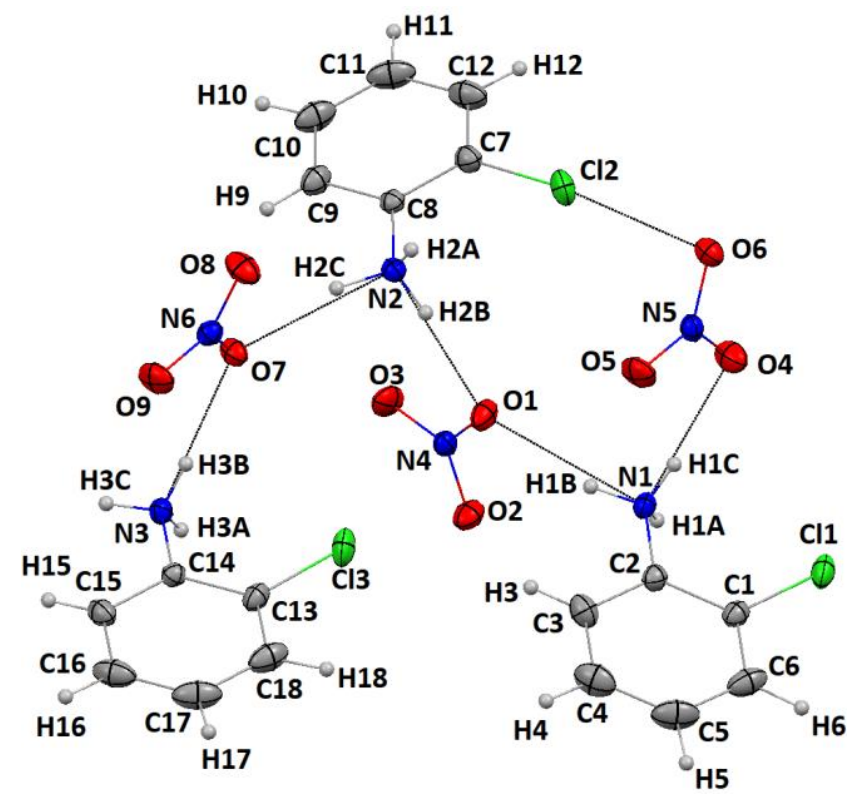

Figure 1. A view of the asymmetric unit $\boldsymbol{o}-\boldsymbol{C l A N}$ with atom numbering scheme. Dashed lines indicate hydrogen bonds and halogen-oxygen interactions.

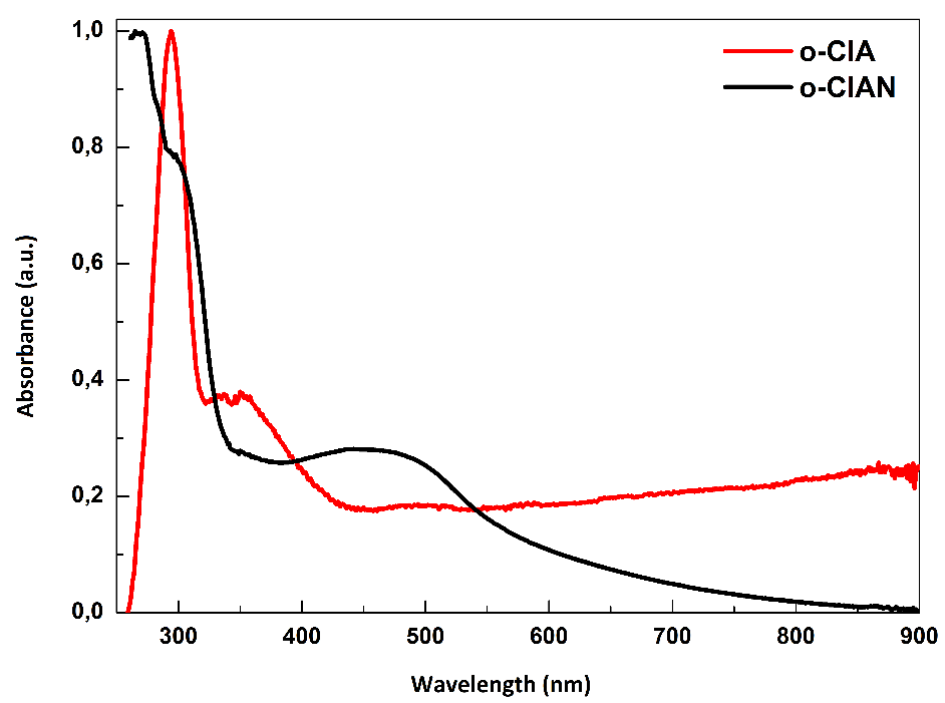

Figure 2. The absorption spectra of o-chloroaniline (o-ClA) and o-ClAN compounds.

\subsection{UV-Visible spectra}

The UV-Vis spectra of o-Chloroaniline and o-Chloroanilinium Nitrate (o-ClAN) samples are shown in Figure 2. The o-Chloroaniline compound has been measured for comparison. The measurements were performed in $10 \mathrm{~mm}$ quartz cell using a solution of low concentration (c $=10^{-5} \mathrm{~mol} / \mathrm{l}$ ) by dissolving the compounds in ethanol. The measurements were recorded at room temperature in the 250-900 $\mathrm{nm}$ range using a Cary 4000 Varian UV-Visible spectrophotometer. The spectra display mainly two absorption maxima at $293 \mathrm{~nm}$ and $350 \mathrm{~nm}$ for o-Chloroaniline and $269 \mathrm{~nm}$ and $450 \mathrm{~nm}$ for the hybrid compound (o-ClAN). The high energy absorption bands 
are assigned to the $\pi \rightarrow \pi^{*}$ transition and are attributed to the aromatic entities, while the lower energy absorption bands are assigned to the $n \rightarrow \pi^{*}$ transition. We observe thus a clear red-shift of the low-energy band when forming the $\boldsymbol{o}-\boldsymbol{C l A N}$ salt. .

\subsection{Nonlinear optical measurement}

Further structural information regarding the polarity of the sample were acquired through nonlinear diffuse reflectometry at room temperature. For more details, see reference (Kijatkin et al., 2017). For characterization, as-synthesized dry $\boldsymbol{o}$-ClAN salt was compressed into a pellet for later irradiation. Using regeneratively amplified and spectrally tunable femtosecond pulses from a Ti:Sapphire laser system with a successive OPA (Astrella HE+, Coherent Inc. and TOPAS Prime, Light Conversion), light was slightly focused onto the sample surface. Incident peak intensities on the order of $10^{14} \mathrm{~W} / \mathrm{m}^{2}$ with a time-averaged power of $59 \mathrm{~mW}$ at $\lambda=1400$ $\mathrm{nm}$ were applied for the generation of efficient second (SHG) and third harmonic emission (THG). Diffusely emitted light was collected at a small angle with a glass fiber and coupled into a spectrograph (IsoPlane SCT320 and PIXIS:2KB/BUV, Princeton Instruments). In order to compare absolute harmonic emission intensities, a second pellet was prepared from $\mathrm{LiNbO}_{3}: \mathrm{Mg}$ nanopowder (henceforth abbreviated to LNO, particle diameter approx. $70 \mathbf{~ n m}$ ) whose nonlinear optical properties have been well-investigated and applied (Staedler et al., 2012., Joulaud et al., 2013; Dutto et al., 2011; Rogov et al., 2015; Bonacina, 2013).

\section{3- Results and discussion}

\subsection{Structure and crystal packing}

The asymmetric unit of $\boldsymbol{o}-\boldsymbol{C l A N}$ consists of three structurally independent ochloroanilinium cations (ClA-1: $\mathrm{C} 1$ to $\mathrm{C} 6, \boldsymbol{C l A}-2$ : $\mathrm{C} 7$ to $\mathrm{C} 12$ and $\boldsymbol{C l A - 3 :} \mathrm{C} 13$ to $\mathrm{C} 18)$ and three nitrate anions (N4-O1 to O3, N5-O4 to O6 and N6-O7 to O9) (Fig. 1). The organic cations and the nitrate anions are interconnected through hydrogen bonds and halogen-oxygen interactions (Fig. 3). This type of interactions has been observed in only one structure containing the ochloroanilinium cation (Balamurugan et al., 2010) among the five structures deposited in the Cambridge Structural Database CSD (ConQuest Version 1.22, 2018) (Allen, 2002) . The crystal packing of $\boldsymbol{o}-\boldsymbol{C l A N}$ is built of anionic zig-zag chains formed by the nitrate anions alternating with the o-chloroanilinium cations stacked layers parallel to the crystallographic $\boldsymbol{c}$-axis (Fig. 4). The three o-chloroanilinium cations are similar and show typical geometrical features to those observed in similar compounds (Bendeif et al., 2005; Boutobba et al., 2010; Dadda et al., 2012; Takouachet et al., 2016). The average $\mathrm{C}-\mathrm{N}$ bond length for the o-chloroanilinium cations (1.456(2) $\AA$ ) (Table 2) is longer compared to that observed for the o-chloroaniline compound (1.375(2) $\AA$ ) (Nayak et al., 2009), indicating that the proton is transferred from the nitric acid to the amino group of the o-chloroaniline moieties. Compared to the o-chloroaniline structure (Nayak et al., 2009), the internal $\mathrm{C}-\mathrm{C}-\mathrm{C}$ angles at the halogen atoms decrease significantly $\left[120.23(2)^{\circ}\right.$ vs $\left.123.3(5)^{\circ}\right]$ and the internal $\mathrm{C}-\mathrm{C}-\mathrm{C}$ angles at ammonium groups increase $\left[120.39(13)^{\circ}\right.$ vs $\left.115.6(5)^{\circ}\right]$. These angular changes are the consequences of the protonation of the amino groups $(\mathrm{N} 1, \mathrm{~N} 2$ and N3) and of the implication of chlorine atoms in relatively short $\mathrm{Cl} \cdots \mathrm{O}$ halogen-oxygen interactions $(\mathrm{Cl} 1 \cdots \mathrm{O} 7=3.087(2) \AA$ and $\mathrm{Cl} 2 \cdots \mathrm{O} 6=$ 
3.084(2) $\AA$ and $\mathrm{Cl} 3 \ldots \mathrm{O} 1=3.288(2) \AA$ ). It is noteworthy that the three cations $(\boldsymbol{C l A} \mathbf{- 1}, \boldsymbol{C l A} \mathbf{- 2}$ and $\boldsymbol{C l A 3}$ ) form through their ammonium groups (N1, N2 and N3) four $\mathrm{N}-\mathrm{H} \cdots \mathrm{O}$ hydrogen bonds with three different $\mathrm{NO}_{3}{ }^{-}$anionic neighbours (Table 3). Such intermolecular interactions produce ring motifs $R_{3}^{5}(16)$ between the organic cations and the nitrate anions (Fig. 4).

Table 2 selected geometric parameters $\left(\AA{ }^{\circ}{ }^{\circ}\right)$

\begin{tabular}{|l|l|l|l|}
\hline $\mathrm{C} 11-\mathrm{C} 1$ & $1.733(2)$ & $\mathrm{N} 4-\mathrm{O} 1$ & $1.278(2)$ \\
\hline $\mathrm{C} 12-\mathrm{C} 7$ & $1.731(2)$ & $\mathrm{N} 4-\mathrm{O} 2$ & $1.248(2)$ \\
\hline $\mathrm{C} 1-\mathrm{C} 13$ & $1.729(2)$ & $\mathrm{N} 4-\mathrm{O} 3$ & $1.227(2)$ \\
\hline $\mathrm{N} 1-\mathrm{C} 2$ & $1.453(2)$ & $\mathrm{N} 5-\mathrm{O} 4$ & $1.261(2)$ \\
\hline $\mathrm{N} 2-\mathrm{C} 8$ & $1.459(2)$ & $\mathrm{N} 5-\mathrm{O} 5$ & $1.231(2)$ \\
\hline $\mathrm{N} 3-\mathrm{C} 14$ & $1.458(2)$ & $\mathrm{N} 5-\mathrm{O} 6$ & $1.263(2)$ \\
\hline $\mathrm{C} 1-\mathrm{C} 2$ & $1.387(3)$ & $\mathrm{N} 6-\mathrm{O} 7$ & $1.276(2)$ \\
\hline $\mathrm{C} 2-\mathrm{C} 3$ & $1.390(3)$ & $\mathrm{N} 6-\mathrm{O} 8$ & $1.250(2)$ \\
\hline $\mathrm{C} 3-\mathrm{C} 4$ & $1.388(3)$ & $\mathrm{N} 6-\mathrm{O} 9$ & $1.230(2)$ \\
\hline $\mathrm{C} 4-\mathrm{C} 5$ & $1.374(5)$ & $\mathrm{C} 12-\mathrm{C} 7$ & $1.390(3)$ \\
\hline $\mathrm{C} 5-\mathrm{C} 6$ & $1.392(4)$ & $\mathrm{C} 13-\mathrm{C} 14$ & $1.385(3)$ \\
\hline $\mathrm{C} 1-\mathrm{C} 6$ & $1.389(3)$ & $\mathrm{C} 14-\mathrm{C} 15$ & $1.388(3)$ \\
\hline $\mathrm{C} 7-\mathrm{C} 8$ & $1.386(3)$ & $\mathrm{C} 15-\mathrm{C} 16$ & $1.391(3)$ \\
\hline $\mathrm{C} 8-\mathrm{C} 9$ & $1.387(3)$ & $\mathrm{C} 16-\mathrm{C} 17$ & $1.382(5)$ \\
\hline $\mathrm{C} 9-\mathrm{C} 10$ & $1.390(3)$ & $\mathrm{C} 17-\mathrm{C} 18$ & $1.386(4)$ \\
\hline $\mathrm{C} 10-\mathrm{C} 11$ & $1.385(4)$ & $\mathrm{C} 18-\mathrm{C} 13$ & $1.390(3)$ \\
\hline $\mathrm{C} 11-\mathrm{C} 12$ & $1.387(4)$ & $\mathrm{C} 1-\mathrm{C} 2-\mathrm{N} 1$ & $119.72(18)$ \\
\hline $\mathrm{C} 2-\mathrm{C} 1-\mathrm{C} 11$ & $119.01(16)$ & $\mathrm{C} 3-\mathrm{C} 2-\mathrm{N} 1$ & $120.17(18)$ \\
\hline $\mathrm{C} 6-\mathrm{C} 1-\mathrm{C} 11$ & $120.45(17)$ & $\mathrm{C} 7-\mathrm{C} 8-\mathrm{N} 2$ & $119.77(17)$ \\
\hline $\mathrm{C} 8-\mathrm{C} 7-\mathrm{C} 12$ & $119.20(15)$ & $\mathrm{C} 9-\mathrm{C} 8-\mathrm{N} 2$ & $119.81(18)$ \\
\hline $\mathrm{C} 12-\mathrm{C} 7-\mathrm{Cl} 2$ & $120.65(18)$ & $\mathrm{C} 13-\mathrm{C} 14-\mathrm{N} 3$ & $119.79(17)$ \\
\hline $\mathrm{C} 14-\mathrm{C} 13-\mathrm{C} 13$ & $119.43(15)$ & $\mathrm{C} 15-\mathrm{C} 14-\mathrm{N} 3$ & $119.60(18)$ \\
\hline $\mathrm{C} 18-\mathrm{C} 13-\mathrm{C} 13$ & $120.56(17)$ & & \\
\cline { 2 - 3 } & & &
\end{tabular}

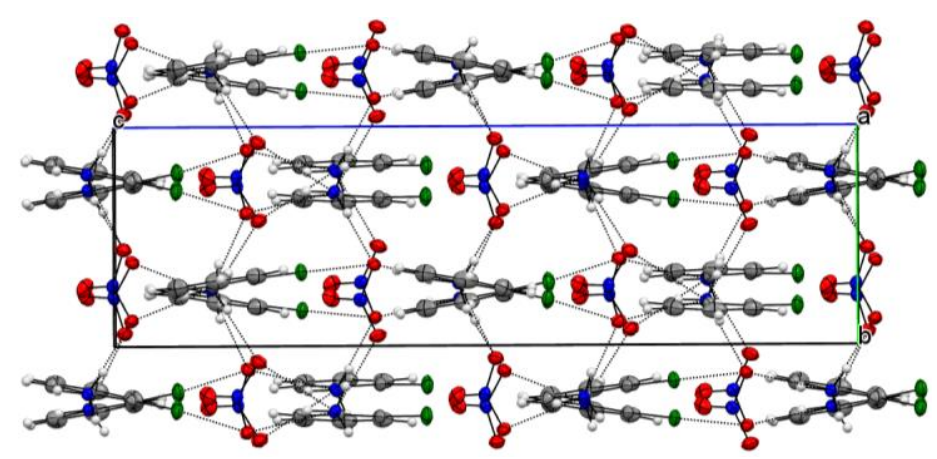

(a)

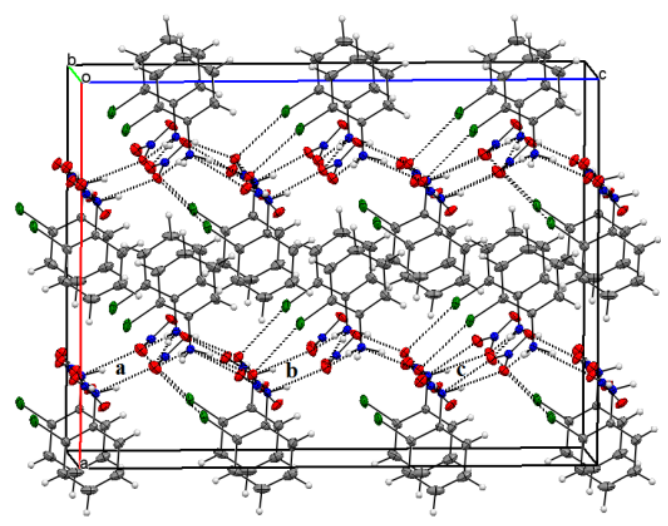

(b)

Figure 3. Projection of the $\boldsymbol{o}-\boldsymbol{C l A N}$ structure along: (a) the crystallographic $\boldsymbol{a}$-axis and (b) the b direction showing a periodic arrangement of anionic zig-zag chains formed by the nitrate anions and ochloroanilinium cationic layers. Dashed lines indicate intermolecular interactions. 
The geometrical features of the three nitrate entities show a distorted $\mathrm{sp} 2$ configuration. The $\mathrm{O}-\mathrm{N}-\mathrm{O}$ angles are relatively similar ranging from $117.41(16)^{\circ}$ to $122.24(16)^{\circ}$. Each $\mathrm{NO}_{3}{ }^{-}$anion is characterized by one shorter $\mathrm{N}-\mathrm{O}$ bond length and two longer ones (see Table 2 for more details). These significant differences in $\mathrm{N}-\mathrm{O}$ distances [ranging from 1.227(2) $\AA$ to $1.278(2) \AA$ ] can be explained by the fact that the longest $\mathrm{N}-\mathrm{O}$ bond lengths (Table 2) correspond to oxygen atoms involved in hydrogen bonds, however the shortest ones (N4-O3, N5-O5 and N6) correspond to those not implicated in any intermolecular interactions with organic cations. This behavior was also reported in a 4-nitroanilinium nitrate structure (Perpétuo et al., 2004).

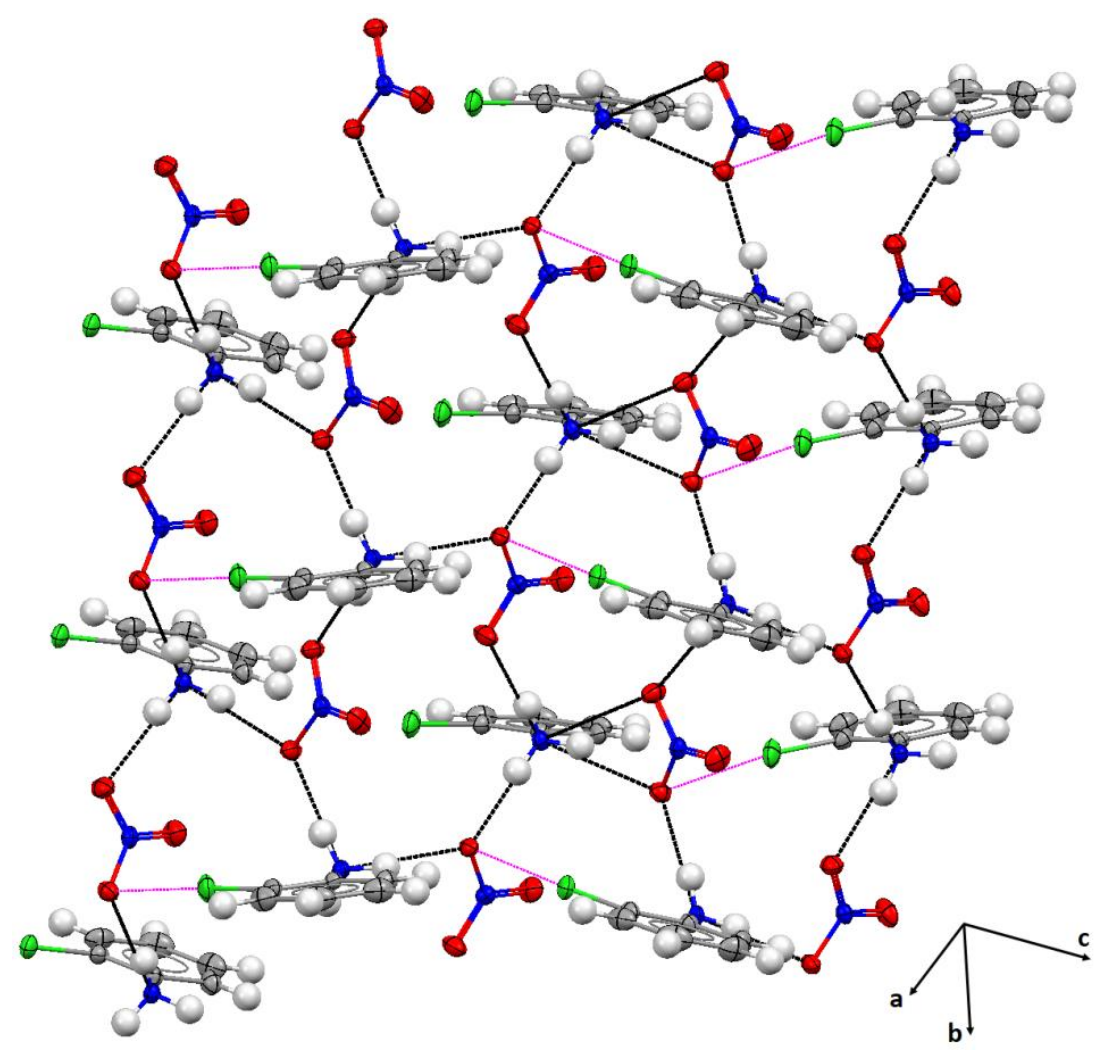

Figure 4. 3D view of the crystal packing of $\boldsymbol{o}-\boldsymbol{C l A N}$, illustrating the formation of ring motifs $R_{3}^{5}(16)$ between organic cations and $\mathrm{NO}_{3}{ }^{-}$anions. $\mathrm{N}-\mathrm{H} \cdots \mathrm{O}$ hydrogen bonds are represented by black dashed line and $\mathrm{Cl} \cdots \mathrm{O}$ interactions are illustrated by magenta dashed line.

\subsection{Molecular Hirshfeld surfaces}

The Hirshfeld surfaces of compound $\boldsymbol{o}$ - $\boldsymbol{C l A N}$ were mapped over $\mathrm{d}_{\text {norm }}(0.5$ to $1.5 \AA)$ and are illustrated in Figure 5. The red circular depressions visible in the front and back surface views indicate hydrogen bonding contacts (Fig. 5). The strongest and shortest interactions between $\mathrm{N}-\mathrm{H}(\mathrm{N} 1-\mathrm{H} 1 \mathrm{~A}, \mathrm{~N} 2-\mathrm{H} 2 \mathrm{~A}, \mathrm{~N} 3-\mathrm{H} 3 \mathrm{~A}$ and $\mathrm{N} 3-\mathrm{H} 3 \mathrm{C})$ and $\mathrm{O}(\mathrm{O} 2, \mathrm{O} 6$ and $\mathrm{O} 8)$ atoms manifest in the Hirshfeld surfaces as the eight (08) bright red areas. The other visible spots on the surfaces correspond to weak $\mathrm{C}-\mathrm{H} \cdots \mathrm{O}$ and $\mathrm{C}-\mathrm{H} \cdots \mathrm{Cl}$ hydrogen bonds. 
Table 3: Hydrogen-bonding geometry $\left(\AA{ }^{\circ}{ }^{\circ}\right)$

\begin{tabular}{|l|l|l|l|l|}
\hline$D-\mathrm{H} \cdots A$ & $D-\mathrm{H}(\AA)$ & $\mathrm{H} \cdots A(\AA)$ & $D \cdots A(\AA)$ & $D-\mathrm{H} \cdots A\left(^{\circ}\right)$ \\
\hline $\mathrm{N} 1-\mathrm{H} 1 \mathrm{C} \cdots \mathrm{O} 4$ & $0.82(3)$ & $1.98(3)$ & $2.800(2)$ & $173(3)$ \\
\hline $\mathrm{N} 1-\mathrm{H} 1 \mathrm{~A} \cdots \mathrm{O} 6^{\mathrm{i}}$ & $0.85(3)$ & $1.99(3)$ & $2.825(2)$ & $169(3)$ \\
\hline $\mathrm{N} 1-\mathrm{H} 1 \mathrm{~B} \cdots \mathrm{O} 1$ & $0.89(3)$ & $1.99(3)$ & $2.858(2)$ & $166(3)$ \\
\hline $\mathrm{N} 1-\mathrm{H} 1 \mathrm{~B} \cdots \mathrm{O} 2$ & $0.89(3)$ & $2.43(3)$ & $3.095(2)$ & $132(2)$ \\
\hline $\mathrm{N} 2-\mathrm{H} 2 \mathrm{~B} \cdots \mathrm{O} 1$ & $0.87(3)$ & $1.93(3)$ & $2.799(2)$ & $174(3)$ \\
\hline $\mathrm{N} 2-\mathrm{H} 2 \mathrm{~A} \cdots \mathrm{O} 2^{\text {iv }}$ & $0.88(3)$ & $1.96(3)$ & $2.821(2)$ & $166(3)$ \\
\hline $\mathrm{N} 2-\mathrm{H} 2 \mathrm{C} \cdots \mathrm{O} 7$ & $0.94(3)$ & $1.90(3)$ & $2.823(2)$ & $166(2)$ \\
\hline $\mathrm{N} 2-\mathrm{H} 2 \mathrm{C} \cdots \mathrm{O} 8$ & $0.94(3)$ & $2.53(3)$ & $3.185(2)$ & $127(2)$ \\
\hline $\mathrm{N} 3-\mathrm{H} 3 \mathrm{~B} \cdots \mathrm{O} 7$ & $0.84(3)$ & $1.95(3)$ & $2.793(2)$ & $175(2)$ \\
\hline $\mathrm{N} 3-\mathrm{H} 3 \mathrm{~A} \cdots \mathrm{O} 8^{\mathrm{i}}$ & $0.87(2)$ & $1.96(3)$ & $2.819(2)$ & $171(3)$ \\
\hline $\mathrm{N} 3-\mathrm{H} 3 \mathrm{C} \cdots 6^{\mathrm{ii}}$ & $0.87(4)$ & $2.08(3)$ & $2.904(2)$ & $159(2)$ \\
\hline $\mathrm{N} 3-\mathrm{H} 3 \mathrm{C} \cdots \mathrm{O} 4^{\mathrm{ii}}$ & $0.87(4)$ & $2.34(3)$ & $3.038(2)$ & $138(2)$ \\
\hline
\end{tabular}

Symmetry code(s): (i) $x, y-1, z$; (ii) $-x+1 / 2, y-1 / 2, z+1 / 2$; (iii) $x, y+1, z$; (iv) $x-1 / 2,-y+3 / 2, z$.

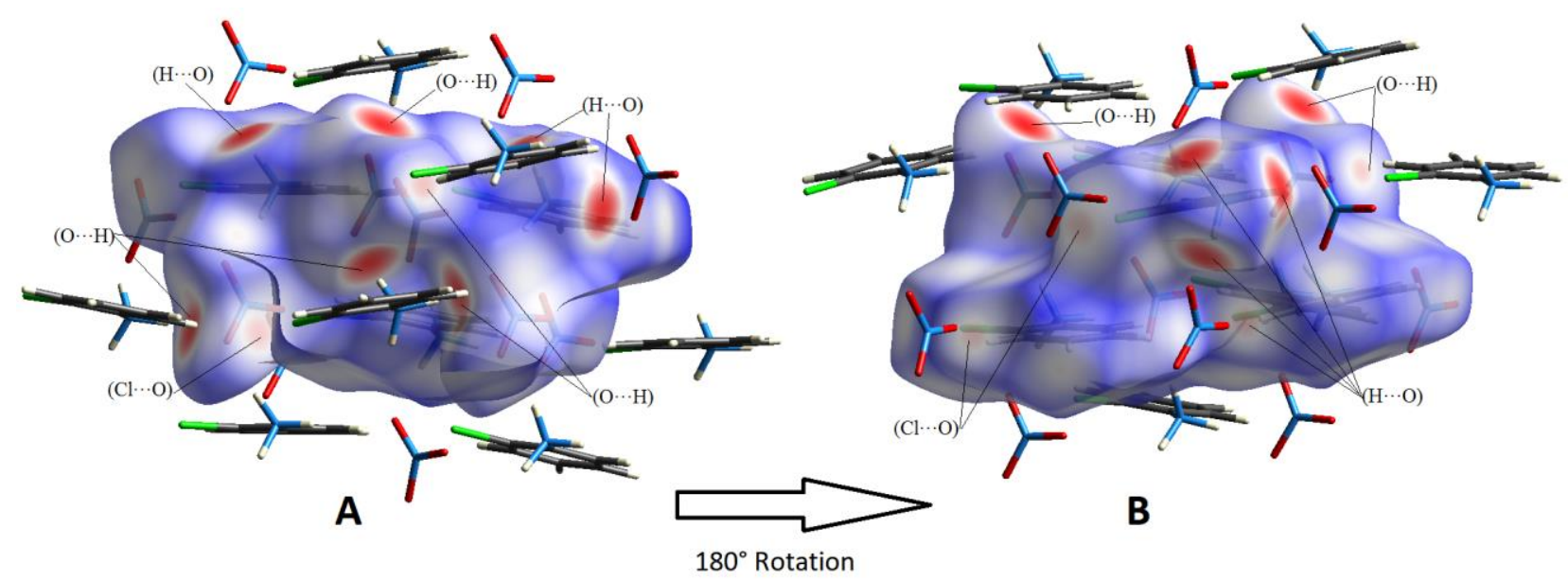

Figure 5. 3D Hirshfeld maps with $d_{\text {norm }}$ in the range 0.5 to $1.5 \AA$ : $\mathbf{A}$ : front view and $\mathbf{B}$ back view. Red circle indicating short hydrogen bonds.

The 2D fingerprint plots are used for identification and separation of intermolecular interactions and relative contribution of these interactions can be obtained from the area of the surfaces. The 2D fingerprint plots of the studied compound are shown in Fig. 6.

The $\mathrm{O} \ldots \mathrm{H} / \mathrm{H} \ldots \mathrm{O}$ contacts, which are attributed to $\mathrm{N}-\mathrm{H}$... O hydrogen-bonding interactions, appear as two sharp symmetric spikes in the two dimensional fingerprint maps (Fig. 6b). They have the most significant contribution to the total Hirshfeld surfaces, accounting for more than 
40.9\%. The presence of these long spikes is characteristic of strong and short hydrogen bonds associated with the cation-anion interactions (Table 3). Moreover, significant contributions to the surfaces are made by the H...C/C...H contacts that appear as symmetrical wings (Fig. 6c) in the fingerprint plots and have a contribution of $19 \%$ of the total $H S$. The H...H contacts appear in the middle of the scattered points in the two-dimensional fingerprint maps (Fig. 6d). They were found to be the third highest contributors towards the Hirshfeld surface, after the $\mathrm{H}$...O/O ...H and H...C/C...H contacts, covering 13.9\%. It is important to note that this analysis reveals a significant contribution of $\mathrm{H}$...Halogen interactions (10.9\%) comparable to that of $\mathrm{H}$...H contacts. The H...Halogen interactions appear as wings on the top left $(\mathrm{H} \ldots \mathrm{Cl})$ and bottom right $(\mathrm{Cl} \ldots \mathrm{H})$ of the $2 \mathrm{D}$ fingerprint plots (Fig. 6e). Apart from the above interactions, the other interactions $(\mathrm{Cl} \ldots \mathrm{C} / \mathrm{C} \ldots \mathrm{Cl}, \mathrm{O} \ldots \mathrm{O}, \mathrm{Cl} \ldots \mathrm{O} / \mathrm{O} \ldots \mathrm{Cl}, \mathrm{N} \ldots \mathrm{H} / \mathrm{H} \ldots \mathrm{N}$ and $\mathrm{C} \ldots \mathrm{C})$ are also observed and are highlighted in Fig. 6f-j. Their contributions are less than 5\% of the total area of the $H S$.

From this analysis, we observe that the major contributions come from the four molecular interactions mentioned above, namely: the O...H/H... , H...C/C...H, H...H and Cl... $\mathrm{H} / \mathrm{H} \ldots \mathrm{Cl}$. These interactions represent $\sim 85 \%$ of the total contributions to the Hirshfeld surfaces and show the diversity of the intermolecular interactions leading to unusual nonlinear optical properties of this molecular compound. It has been also shown that the efficiency of the hydrogen-bond interactions play a key role in the significant enhancement of NLO properties compared to similar compounds ((Bouchouit et al., 2010; Sajana et al., 2010; Tanak et al., 2014). 


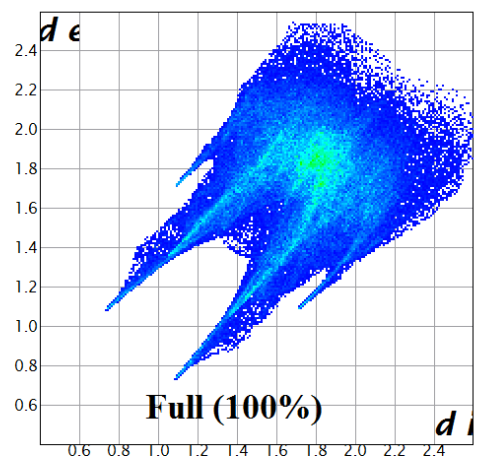

a

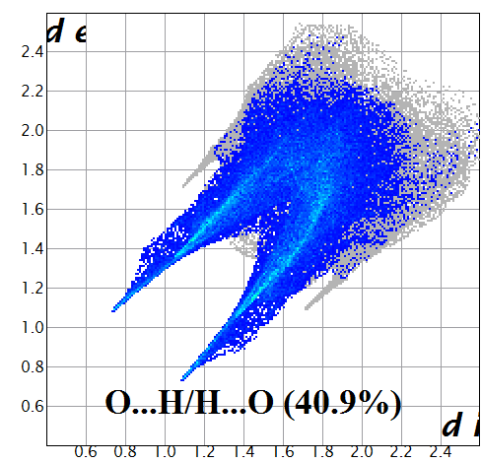

b

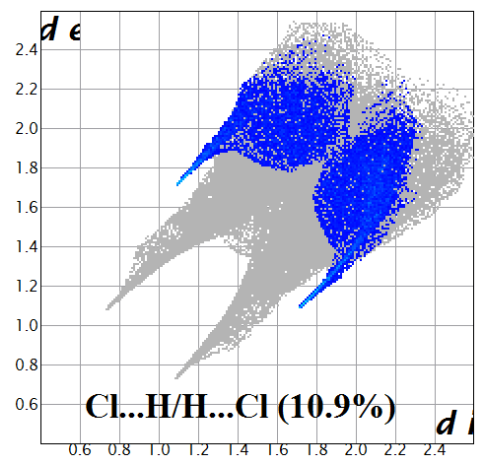

e

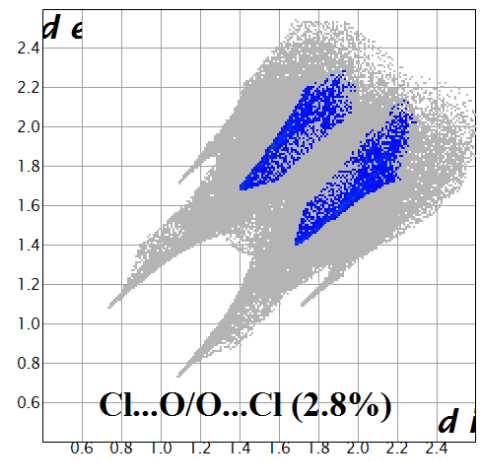

h

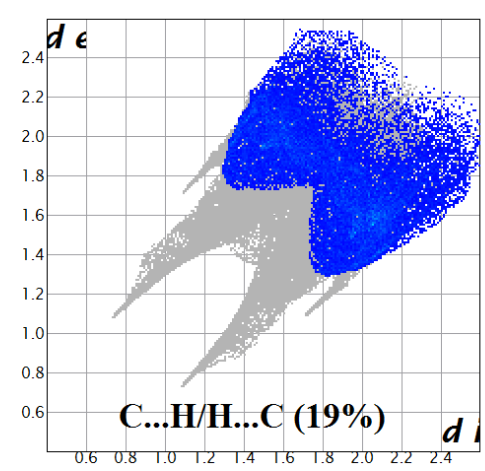

c

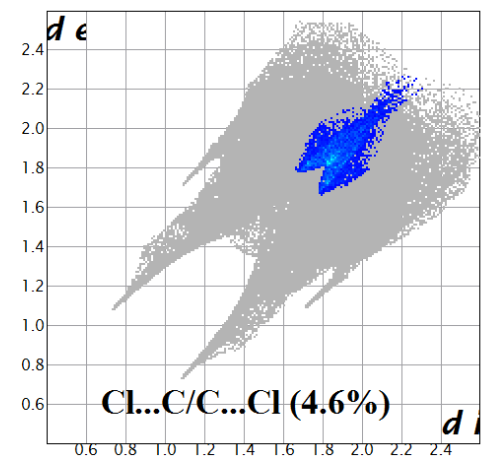

f

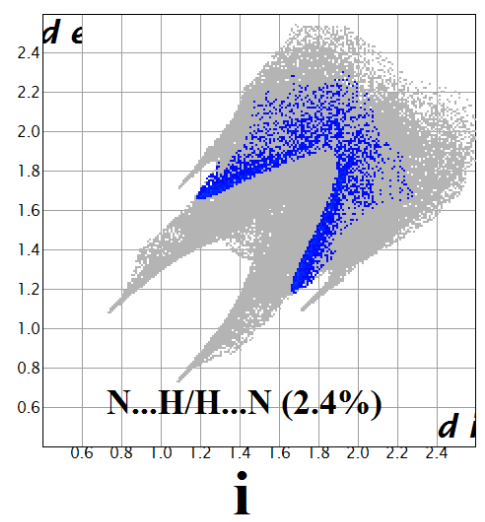

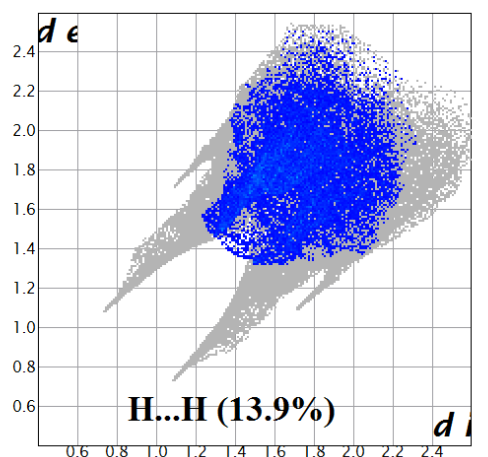

d

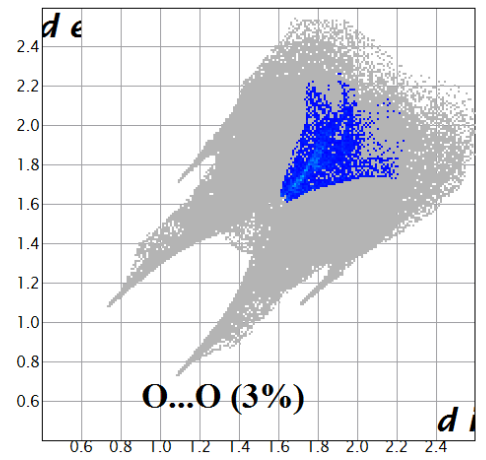

g

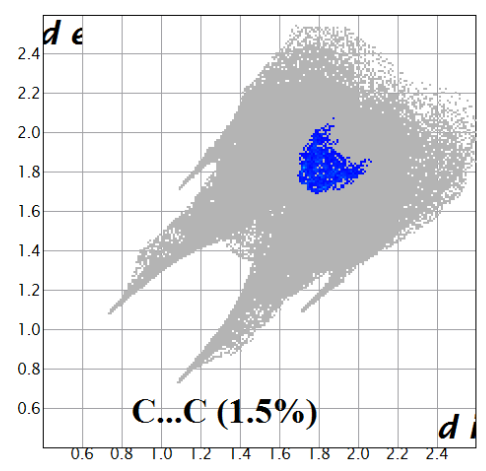

j

Figure 6. 2D fingerprint plots of $\boldsymbol{o - C l A N}$ compound for different intermolecular interactions. 


\subsection{NLO properties}

Upon irradiation with femtosecond pulses (Incident peak intensities on the order of $1014 \mathrm{~W} / \mathrm{m}^{2}$ with a time-averaged power of $59 \mathrm{~mW}$ at $\lambda=1400 \mathrm{~nm}$ ), $\boldsymbol{o}$ - $\boldsymbol{C l A N}$ hybrid crystal exhibit notable second and third harmonic generation at wavelengths of $700 \mathrm{~nm}$ and $467 \mathrm{~nm}$, respectively. This is underlined by the measured harmonic ratio $f_{R}$ (see reference (Kijatkin et al., 2017) for the definition of $f_{R}$ ) ranging up to $5.6 \times 10^{7}$ which approaches that of LNO being $1.0 \times 10^{9}$. This finding has two consequences: 1 ) because $f_{R}$ is well above unity, this measurement confirms the polarity of the o-ClAN compound (Kijatkin et al., 2017), generated by the different intermolecular interactions discussed above and 2) the THG signal of $\boldsymbol{o}-\boldsymbol{C l} \boldsymbol{A} \boldsymbol{N}$ normalized to its SHG counterpart is more pronounced than for LNO. Visual inspection confirms this finding: whereas LNO irradiated with a wavelength $\lambda=1400 \mathrm{~nm}$ appears in a red-magenta hue, $\boldsymbol{o}-\boldsymbol{C l A N}$ incorporates relatively larger cyan components at $467 \mathrm{~nm}$, thereby appearing in more blue hue. However, the nonlinear optical signal of the $\boldsymbol{o}-\boldsymbol{C l} \boldsymbol{A} \boldsymbol{N}$ compound is highly time-dependent and deteriorates significantly upon prolonged exposure (Fig. 7).
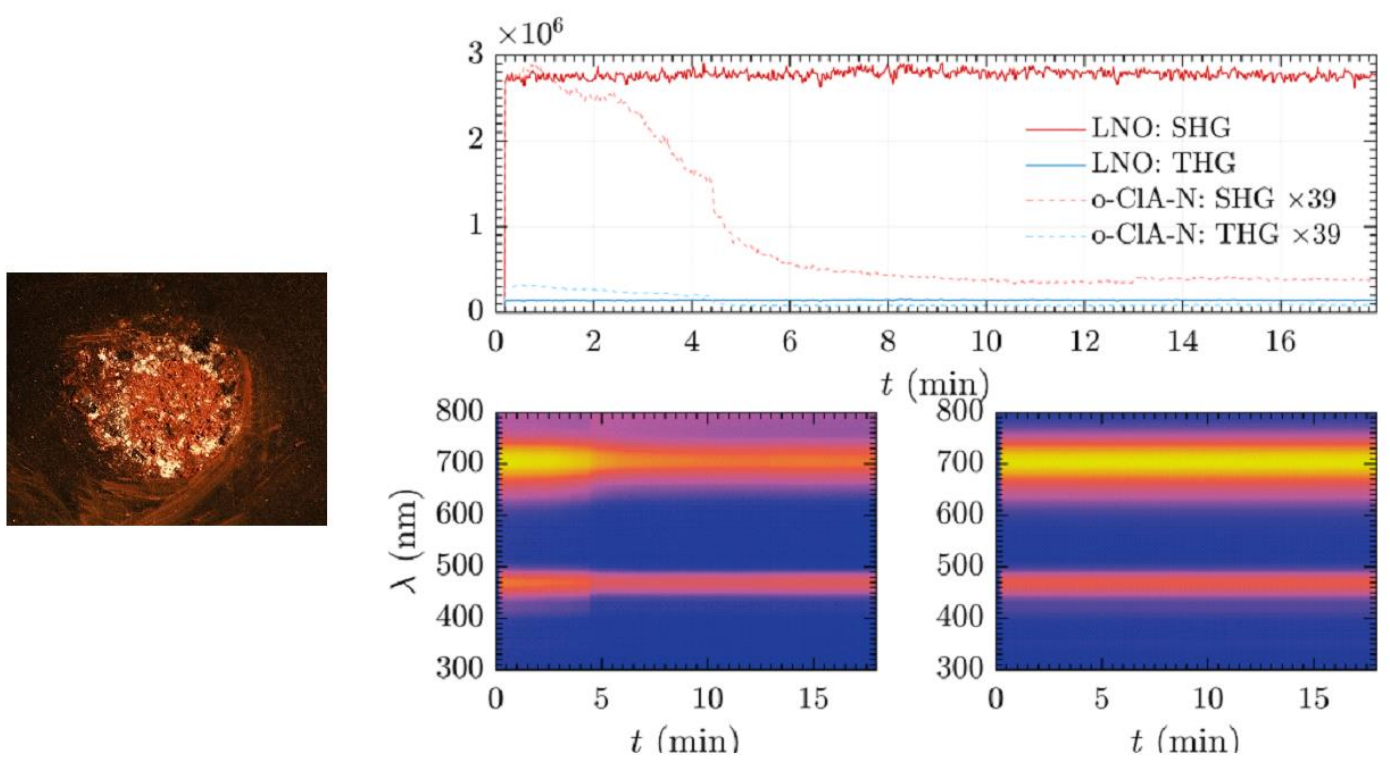

Figure 7. Left: $\boldsymbol{o}-\boldsymbol{C l A N}$-pellet before exposure. The pellet measures $5 \mathrm{~mm}$ in diameter. Right top: second (red) and third (blue) harmonic emission from LNO (solid lines) and $\boldsymbol{o}-\boldsymbol{C l A N}$ (dashed lines) as a function of time. SHG and THG signal of $\boldsymbol{o}$-CIAN have been scaled for better visibility. Right bottom: normalized spectral traces for $\boldsymbol{o}$-ClAN (left) and LNO (right) scaling from yellow (high) to blue (low signal).

During exposure, thermal damage of $\boldsymbol{o}-\boldsymbol{C l A N}$ was observed at the irradiated spot which is the like cause for the time-dependent decline of harmonic signals. In contrast, LNO showed neither signal nor sample degradation over time. Moving the excitation spot on the o-ClAN-pellet surface showed significant fluctuations in the overall emitted nonlinear signal. This can be related to the inhomogeneous surface of the o-ClAN-sample (Fig. 7, left) which is only selectively illuminated. 
Additional measurements at different positions on the sample revealed that the $\boldsymbol{o}-\boldsymbol{C l A N}$ compound can occasionally challenge harmonic emission of polar materials for short timescales. In terms of the emitted SHG-signal at an incident wavelength $\lambda=1400 \mathrm{~nm}, \boldsymbol{o}-\boldsymbol{C l A N}$ typically ranges between $1 / 10$ and $1 / 300$ of the conversion efficiency of the LNOnanocrystalline powder sample.

\section{Conclusion}

In summary, we have reported in this work the synthesis, structural characterization and nonlinear optical (NLO) properties of anilinium organic-inorganic crystal of formula $\left[\left(\mathrm{C}_{6} \mathrm{H}_{7} \mathrm{NCl}^{+}, \mathrm{NO}_{3}\right)_{3}\right](\boldsymbol{o}-\mathrm{ClAN})$. The elaboration of this material was driven by the association of two molecular entities characterized by an electronic configuration favorable to charge transfer allowing therefore high polarizability expected to yield enhanced NLO properties.

The accurate structural analysis performed at low temperature $(100 \mathrm{~K})$ shows that the crystal structure can be described in the pyroelectric crystalline symmetry class. The Hirshfeld surfaces analysis coupled to the structural investigation reveal that crystal packing is characterized by a three-dimensional network of hydrogen bonds and the main contributions are provided by the $\mathrm{O} \ldots \mathrm{H}, \mathrm{C} \ldots \mathrm{H}, \mathrm{H} \ldots \mathrm{H}$ and $\mathrm{Cl} . . \mathrm{H}$ interactions, which alone represent $\sim 85 \%$ of the total contributions to the Hirshfeld surfaces. The different intermolecular interactions acting in accordance with the significant and directional $\mathrm{H}$...H and $\mathrm{H}$...Halogen contacts are responsible for steering molecules in the non-centrosymmetric space group leading to interesting NLO properties. The NLO measurements shows that the $\boldsymbol{o}-\boldsymbol{C l A N}$ exhibits remarkable second (SHG) and third (THG) harmonic generation that confirms its polarity driven by the different intermolecular interactions. The obtained results were also compared to those of the reference material $\mathrm{LiNbO}_{3}$ and show that THG to SHG ratio of the studied compound is more pronounced than for $\mathrm{LiNbO}_{3}$.

\section{Acknowledgements}

This work was supported by the Universite de Lorraine, the CNRS and PHC PROCOPE 40539XA, which are gratefully acknowledged. H.A. is indebted to the Algerian Ministry of Research and Université Abbes Laghrour-Khenchela for a doctoral fellowship. The authors would like to thank the Deutsche Forschungsgemeinschaft (DFG INST 190/165-1 FUGG) and German Academic Exchange Service (DAAD 57139940) for financial support. The authors like to thank Laura Oláh and Zsuzsanna Szaller from the Wigner Research Center for Physics, Budapest, for the preparation of LNO nanopowders within the project MÖB 65056 of the Hungarian Tempus Foundation. 


\section{References}

Allen, F.H. (2002). Acta Crystallogr. B58, 380-388.

Balamurugan, P., Jagan, R. \& Sivakumar, K., (2010). Acta Crystallogr. C66, o109-o113.

Bayar, I., Khedhiri, L., Jeanneau, E., Lefebvre, F \& Ben Nasr, C. (2017). Journal of Molecular Structure. 1137, 373-379.

Below, H., Lehan, N \& Kramer, A. (2004). Microchim, Acta .146. 129-135.

Bendeif, E.-E., Dahaoui, S., François, M., Benali-Cherif, N \& Lecomte, C. (2005). Acta Crystallogr. B61, 700-709.

Bonacina, L. (2013). Mol. Pharmaceutics. 10(3), 783-792

Bouchouit, K., Bendeif, E.-E., EL Ouazzani, H., Dahaoui, S., Lecomte, C., Benali-Cherif, N \& Sahraoui, B. (2010). Chemical Physics. 375, 1-7.

Boutobba, Z., Direm, A \& Benali-Cherif, N. (2010). Acta Crystallogr. E66, o595-o596.

Cao, L; Z. Liu, T. Wang, H. Dai, L. Zhang, X. Tao \& D. Cui. (2012). CrystEngComm. 14, 5795-5800.

Dadda, N., Nassour, A., Guillot, B., Benali-Cherif, N \& Jelsch, C. (2012). Acta Crystallogr. A68, 452463.

Dutto, F., Raillon, C., Schenk, K. \& Radenovic, A. (2011). Nano Letters. 11(6), 2517-2521

Farrugia, L. J. (1999). J. Appl. Crystallogr. 32, 837-838.

Hirshfeld, E. L. (1977). Theor. Chim. Acta. 44, 129-138

Joulaud, C., Mugnier, Y., Gnon Djanta, Y., Dubled, M., Marty, J-C., Galez, C., Wolf, -P., Bonacina, L \& Le Dantec, R. (2013). Journal of Nanobiotechnology. 11(Suppl 1):S8.1-9.

Kijatkin, C., Eggert, J., Bock, S., Berben, D., Oláh, L., Szaller, Z., Kis, Z. \& Imlau, M. (2017). Photonics. 4, 11-24.

Nayak, S. K., Prathapa, S. J. \& Row, T.N.G., (2009). Journal of Molecular Structure. 935, 156-160.

Perpétuo, G. J \& Janczak, J. (2004). Acta Crystallogr. C60, o768-o770.

Rigaku Oxford Diffraction. (2017) CrysAlis CCD and CrysAlis RED (Versions 1.171.38.46), Rigaku Oxford Diffraction. Yarnton, England

Rogov, A., Mugnier, Y \& Bonacina, L., (2015). J. Opt. 17, 033001- 033012

Sajana, D., Ravindra, H.J., Neeraj Misra \& Hubert Joe, I. (2010). Vibrational Spectroscopy. 54, 72-80. Sheldrick, G. M. (2008). Acta Cryst. A64, 112-122.

Spackman, M. A \& Jayatilaka, D. (2009). CrystEngComm. 11, 19-32.

Staedler, D., Magouroux, T., Hadji, R., Joulaud, C., Extermann, J., Schwung, S., Passemard, S., Kasparian, C., Clarke, G., Gerrmann, M., Le Dantec, R., Mugnier, Y., Rytz, D., Ciepielewski, D., Galez, C., Gerber-Lemaire, S., Juillerat-Jeanneret, L., Bonacina, L \& Wolf, J-P. (2012). ACS Nano. 6(3), 25422549.

Takouachet, R., Benali-Cherif, R., Bendeif, E.-E., Benali-Cherif, N., Pillet, S \& Schaniel, D. (2016). Inorganica Chimica Acta. 446, 6-12.

Tanak, H., Pawlus, K., Marchewka, M.K \& Pietraszko, A. (2014). Spectrochimica Acta Part A: Molecular and Biomolecular Spectroscopy. 118, 82-93.

Wang, J. J., Wang, Y. Q., Cao, F. F., Guo, Y. G \& Wan, L. J. (2010). J. Am. Chem. Soc. 132, $12218-$ 12221.

Wolff, S. K., Grimwood, D. J., McKinnon, J. J., Turner, M. J., Jayatilaka, D. \& Spackman, M. A. (2012). CrystalExplorer, Version 3.1. Perth: University of Western Australia.

Yunnikova, L. P., Akent'eva, T. A \& Aleksandrova, G. A. (2013). Pharm. Chem. J. 46, 27-29. 